\title{
Noninvasive ventilation in chronic obstructive pulmonary disease, bronchiectasis and cystic fibrosis
}

\author{
J.A. Wedzicha*, J-F. Muir ${ }^{\#}$
}

\begin{abstract}
Noninvasive ventilation in chronic obstructive pulmonary disease, bronchiectasis and cystic fibrosis. J.A. Wedzicha, J-F. Muir. C ERS Journals Ltd 2002.

ABSTRACT: Although long-term oxygen therapy (LTOT) improves survival, it has little effect on hypoventilation and other outcomes in patients with hypercapnic respiratory failure due to chronic obstructive pulmonary disease (COPD).

Recent studies have shown that the use of noninvasive positive-pressure ventilation, when used in combination with LTOT in selected stable COPD patients, controls hypoventilation and improves daytime arterial blood gases, sleep quality, health status and may have a benefit in reducing exacerbation frequency and severity. Patients who show the greatest reduction in overnight carbon dioxide tension in arterial blood with ventilation are most likely to benefit from long-term ventilatory support.

Some benefits have also been shown in patients with chronic respiratory failure due to bronchiectasis and cystic fibrosis, though survival is inferior in this patient group.

As most studies of noninvasive positive-pressure ventilation in chronic obstructive pulmonary disease have been relatively short term, large multicentre studies with survival, exacerbations and hospital admissions as the primary end points are required to evaluate longer term effects.

Eur Respir J 2002; 20: 777-784.
\end{abstract}

*St Bartholomew's Hospital, London,
UK. "University Hospital, Rouen, France.

Correspondence: J.A. Wedzicha, Academic Respiratory Medicine, Dominion House, St Bartholomew's Hospital, London, EC1A 7BE, UK.

Fax: 442076018616

E-mail J.A.Wedzicha@qmul.ac.uk

Keywords: Bronchiectasis

chronic obstructive pulmonary disease cystic fibrosis

nocturnal hypoventilation,

noninvasive ventilation

Received: June 52001

Accepted after revision: January 14 2002
Domiciliary long-term oxygen therapy (LTOT) is one of the few interventions in patients with chronic obstructive pulmonary disease (COPD) that improves survival when used for $\geqslant 15 \mathrm{~h} \cdot \mathrm{day}^{-1}[1,2]$. LTOT has also been shown to have a number of important physiological benefits, although it has little effect on other factors including health-related quality of life or reduction in disease exacerbation. Many of these COPD patients exhibit marked abnormalities of overnight ventilation, with a consequent derangement of blood gases and ventilatory muscle dysfunction, which may contribute to the progressive deterioration seen in this group [3-5]. It has therefore been suggested that ventilatory support may confer further advantages when used in addition to LTOT.

Early studies in patients with respiratory failure due to COPD, using mainly negative-pressure techniques, have largely demonstrated no benefit of ventilation [6-8]. However, early uncontrolled studies in COPD patients on noninvasive positive-pressure ventilation (NPPV), mainly using nasal masks, showed that it was possible to apply ventilation to these patients, as had been previously found in chest wall and neuromuscular disease. The addition of NPPV was shown to produce significant improvements in arterial blood gases and control of nocturnal hypoventilation [9-11].

\section{Chronic obstructive pulmonary disease}

\section{Physiological considerations}

In patients with COPD, ventilation is reduced during sleep compared to wakefulness, with further reduction in ventilation during rapid eye movement sleep. This fall in ventilation is largely due to a reduction in tidal volume and is not usually associated with apnoea [12]. The hypoventilation will cause considerable arterial oxygen desaturation at night, which may be an important factor in the development of chronic pulmonary hypertension [4-5]. During sleep there is a reduction in carbon dioxide $\left(\mathrm{CO}_{2}\right)$ responsiveness [13] and an increase in upper airway resistance, leading to further respiratory insufficiency

Previous articles in this series: No. 1: Brochard L, Mancebo J, Elliott MW. Noninvasive ventilation for acute respiratory failure. Eur Respir J 2002; 19: 712-721. No. 2: Ferrer M, Bernadich O, Nava S, Torres A. Noninvasive ventilation after intubation and mechanical ventilation. Eur Respir J 2002; 19: 959-965. No 3: Elliott MW, Confalonieri M, Nava S. Where to perform noninvasive ventilation? Eur Respir J 2002; 19: 1159-1166. No 4: Corrado A, Gorini M. Negative-pressure ventilation: is there still a role? Eur Respir J 2002; 20: 187-197. No 5: Shneerson JM, Simonds AK. Noninvasive ventilation for chest wall and neuromuscular disorders. Eur Respir J 2002; 20 : $480-487$. 
[14]. Other mechanisms for the arterial oxygen desaturation include a higher physiological dead space in patients with COPD, effects of hyperinflation and ventilation/perfusion inequalities.

Nocturnal arterial oxygen desaturation can be corrected with supplemental oxygen therapy, although this may be associated with worsening hypercapnia especially in the early hours of the morning [15], and little effect is observed on daytime blood gases. A study of patients with stable COPD by means of sequential polysomnography and overnight blood gas monitoring noted a relationship between the fall in arterial oxygen saturation overnight and progression of the underlying disease, particularly in those patients with concomitant hypercapnia [16]. The deterioration in hypoventilation and hypercapnia may further impair diaphragmatic function [17] and respiratory mechanics and eventually have a deleterious effect on central respiratory drive.

It has been suggested that in COPD the respiratory muscles are subject to fatigue, and that improvements in lung mechanics and a consequent reduction in workload may facilitate muscle rest and relief of fatigue. Several investigators have shown that the application of noninvasive ventilation, using both positive- and negative-pressure techniques, may produce significant reductions in diaphragmatic electromyogram (EMG) activity and work of breathing [7, 18-20]. However, no long-term controlled study has yet shown an improvement in measures of ventilatory muscle strength with the addition of assisted ventilation.

Evidence is emerging that the mechanisms of reversal of the hypoventilation and thus improvement of arterial blood gases during the daytime is an improvement in $\mathrm{CO}_{2}$ responsiveness. HILl et al. [21] showed that withdrawal of noninvasive ventilation for a period of 1 week in patients with chest wall and neuromuscular disease produced deterioration in patients' symptoms and nocturnal hypoventilation, but no effects were seen on respiratory muscle function. Similar results were reported by GoLDSTEIN et al. [22] in patients with neuromuscular disease, suggesting that the application of noninvasive ventilation improves the sensitivity to $\mathrm{CO}_{2}$. ElliotT et al. [23], in a study of nocturnal nasal ventilation in patients with COPD, showed that those patients who improved their blood gases had associated increased chemosensitivity to $\mathrm{CO}_{2}$. However, they were unable to demonstrate any effects on respiratory muscle fatigue with the addition of NPPV.

Patients with hypoxic COPD also have sleep disruption, although the effects of supplemental oxygen therapy on sleep quality have been variable, with some studies showing benefit and others not documenting any effect $[15,24]$. Although some of these patients may have coincident sleep apnoea, sleep disruption in COPD is not usually caused by the effects of upper airway obstruction. Patients with COPD may be seen to arouse during episodes of oxygen desaturation, although sleep disruption is also present in patients with COPD who do not desaturate at night [25]. Hypercapnia may be worsened overnight with the addition of supplemental oxygen therapy. An acute elevation of $\mathrm{CO}_{2}$ tension in arterial blood $\left(\mathrm{Pa}_{\mathrm{a}}, \mathrm{CO}_{2}\right)$ during the night may contribute to arousals and thus impaired sleep quality [26]. Control of nocturnal hypercapnia through nasal ventilation may improve sleep quality and thus result in increased neuropsychological performance and quality of life.

\section{Positive pressure via tracheostomy}

Positive-pressure ventilation through a tracheostomy has been widely used in France, although controlled studies of its efficacy in patients with COPD are lacking. Robert et al. [27] showed that patients with COPD had a worse prognosis than in other conditions requiring assisted ventilation such as kyphoscoliosis or in sequelae of tuberculosis. The survival in their COPD group treated by tracheostomy was similar to that of the British Medical Research Council (MRC) long-term oxygen trial [2]. However, a further multicentre study from France of 259 COPD patients showed an improved survival, compared to that of the MRC group for the first 4 yrs and then the survival curves became similar [28]. The suggestion was made that assisted ventilation in COPD should be used at an earlier stage in the natural history of the condition to obtain increased benefit.

\section{Negative pressure}

Negative-pressure ventilation, although effective in patients with chest wall and neuromuscular disease, has been less successful in patients with COPD, mainly due to poor patient tolerance and compliance with the technique. Musculo-skeletal and chest discomfort are common with negative-pressure devices and the equipment tends to be more cumbersome. Upper airway obstruction may also be potentiated with the use of negative-pressure equipment.

A randomised crossover study comparing the Poncho-wrap negative ventilator with standard care in 20 stable COPD outpatients over 6 months, showed no improvement in blood gases, maximal inspiratory and expiratory pressures or exercise tolerance [8]. However, 11 of the 20 patients withdrew from the study because of inability to tolerate the ventilator. Almost all the patients were dissatisfied with the treatment and used it for $4.1 \mathrm{~h}$ daily, considerably less time than recommended. Patients most frequently complained of musculo-skeletal pain and inconvenience.

A further randomised study of negative-pressure ventilation in COPD using the Pulmo-wrap device was reported in hospital patients [6]. The trial, involving 16 patients with COPD, aimed to determine whether, negative-pressure ventilation, when added to a standard exercise training and pulmonary rehabilitation programme, improved ventilatory muscle function, perception of well-being and exercise performance. There was no physiological advantage documented from the use of negative-pressure ventilation and again compliance with the equipment was poor. 
However, as in the previous study described, the patients with COPD had only modest hypercapnia.

SHAPIRO et al. [7] in Montreal reported a study in 184 patients with COPD, who were randomised to receive either active negative-pressure ventilation or sham treatment over a 12 week period of home use. There were no significant changes in the active compared to the sham group with respect to the outcome measures, which included cycle exercise endurance time, severity of dyspnoea, quality of life, arterial blood gas tensions and respiratory muscle strength. The authors concluded that negative-pressure ventilation was ineffective as a method for resting respiratory muscles in patients with stable COPD. However, compliance with the technique was poor once again, with 63 patients either not using the respirators at all, or stopping before completing the 12 -week study period. Although the aim of the study was for the patients to use the ventilation overnight, this was unsuccessful and most of the patients were only treated during the daytime, with the vast majority receiving $<3.5 \mathrm{~h}$ of ventilation daily. The aim of the study was to obtain at least a $50 \%$ reduction in the amplitude of the diaphragmatic EMG signal, with an average ventilator use of $5 \mathrm{~h}$ daily, but this objective was only achieved in $29 \%$ of the patients.

Other studies in COPD patients have been reported using negative-pressure ventilation on an intermittent basis in hospital. One study showed improvement in blood gases, though the design was uncontrolled, with a small numbers of patients [29]. However, in this study, the patients were significantly hypercapnic. Another controlled study suggested a beneficial effect on ventilatory muscle function in those patients who received negative-pressure ventilation for 3-6 h daily for 3 consecutive days, though no longer term followup or therapy with negative pressure was available [30].

\section{Noninvasive positive-pressure ventilation}

Following the success of NPPV in correcting hypoventilation and daytime blood gases in chest wall and neuromuscular disease, treatment was extended to patients with COPD. Early uncontrolled studies showed that arterial blood gases improved in patients treated with NPPV, with improvement in sleep quality $[9,10,31]$. Results were more favourable compared to those achieved previously with negative-pressure ventilation. However, in these studies compliance with ventilation was not as good as in patients with restrictive chest disease. Patients who showed benefit were the patients who also had significant daytime hypercapnia and whose nocturnal hypercapnia could be successfully reduced by overnight ventilation [10]. Supplemental oxygen was not used routinely with the NPPV in some early studies. This may have reduced benefit because overnight oxygen saturation levels were not adequately corrected with NPPV alone [18].

STRUMPF et al. [32] performed a randomised crossover study of nasal ventilation using a bilevel ventilator in 19 patients with COPD. Compliance proved to be a major problem in the study and only seven patients completed both arms of the protocol; the poor compliance mainly resulting from problems with the nasal/mask interface. This study showed no changes in pulmonary function, respiratory muscle strength, gas exchange, exercise endurance, sleep efficiency, sleep quality, oxygenation or dyspnoea ratings between the two arms of the trial. The only improvements observed were in neuropsychological function. Although these patients had severe airflow obstruction, they showed less severe derangement of blood gases and in particular lesser degrees of hypercapnia, with some patients in the normocapnic range.

Meecham Jones et al. [11] have recently reported a randomised crossover study of nasal pressure support ventilation plus oxygen therapy, compared to domiciliary oxygen therapy alone in 18 hypercapnic COPD patients. The aim of this study was to investigate the effect of the addition of NPPV to patients already established on LTOT. Following a run-in period, each patient received NPPV plus oxygen and oxygen alone in random order for 3 -month periods. There were significant improvements in daytime arterial blood gases, with the mean oxygen tension in arterial blood $\left(\mathrm{Pa}_{\mathrm{a}} \mathrm{O}_{2}\right)$ rising from $5.9 \mathrm{kPa}(44.3 \mathrm{mmHg})$ after the oxygen period to $6.7 \mathrm{kPa}(50.2 \mathrm{mmHg})$ after the addition of NPPV and $\mathrm{Pa}, \mathrm{CO}_{2}$ falling from $7.6 \mathrm{kPa}(57.0 \mathrm{mmHg})$ to $7.0 \mathrm{kPa}(52.5 \mathrm{mmHg})$. There were also improvements seen after the addition of NPPV in overnight $P \mathrm{a}, \mathrm{CO}_{2}$, total sleep time and sleep efficiency, suggesting that control of hypoventilation in these patients is effective and leads to improved sleep quality. The improvement in daytime blood gas values was correlated with the change in overnight $\mathrm{Pa}_{\mathrm{a}} \mathrm{CO}_{2}$. This suggests that the patients who showed the greatest improvement in $P$ a, $\mathrm{CO}_{2}$ with nocturnal nasal ventilation are likely to gain the greatest benefit from the treatment.

Compliance in this study was much better, compared to most previous studies of noninvasive ventilation in COPD, as 14 of the 18 patients completed the study and only one of the patients was withdrawn because of inability to tolerate the equipment. Previous studies had mainly initiated NPPV in outpatients [32], while in this study all patients had nasal ventilation initiated as inpatients, allowing them maximum support and education while they became familiar with the equipment. Improved compliance may also have been due to the greater degree of hypercapnia present in these patients and consequent benefit achieved with ventilation.

As progressive COPD is associated with significant disturbances in activities of daily living, measures of quality of life are essential for assessing the effectiveness of therapies [33]. Studies of LTOT have generally not demonstrated any benefit with respect to quality of life [34]. MeEcham Jones et al. [11] measured quality of life in their study using the St George's Respiratory Questionnaire (SGRQ), which provides a disease-specific measure of quality of life, with three component scores (symptoms, activities and impacts) and a total score [35]. The combination of NPPV with oxygen therapy produced significant improvements in symptom, impact and total quality of life scores as 
compared to oxygen alone. There was no change in the activity component in the two study groups and no change in 6-min walking distance after ventilation. A study involving patients with restrictive ventilatory disorders showed that quality of life was related to sleep quality [36]. Although these findings were not confirmed in the study by МеЕснам Jones et al. [11], it is possible that improvements in sleep time and efficiency contribute to parallel improvements in health-related quality of life. SEemungal et al. [37] have recently shown that quality of life, as assessed using the SGRQ, is strongly related to exacerbation frequency. It is possible that the improvement in health status observed reflects reduction in exacerbation frequency, although this was not addressed in this particular study. NPPV may also protect the patient against deterioration in blood gases during an exacerbation, and this may be the mechanism of any observed survival benefit.

PERRIN et al. [38] followed 14 patients with hypercapnic COPD over a 6-month period and found similar improvements in daytime arterial blood gases. This study is also of interest in that improvements were also found in the total SGRQ score and in the impacts component of the SGRQ score. Another small study investigated the effects of the addition of NPPV to oxygen therapy in severe COPD, but found no significant benefit of NPPV after only 2 weeks of therapy [39]. However, acclimatisation to the ventilator may take longer than a 2-week period and thus the study time was too short. In this study, patients had problems with higher levels of inspiratory positive airway pressure $(\geqslant 15 \mathrm{~cm})$ and this suggests that they required a longer period of acclimatisation to the ventilator.

There is some data on longer term outcome with NPPV in COPD, although this is mainly from uncontrolled studies. LEGER et al. [40] followed 50 COPD patients on NPPV and showed a significant decrease in $\mathrm{Pa}_{\mathrm{a}} \mathrm{CO}_{2}$ in long-term survivors over $2 \mathrm{yrs}$, again emphasising the importance of control of hypoventilation in outcome of NPPV. Probability of continuing nasal ventilation was lower in patients with COPD at $56 \%$, compared to patients with kyphoscoliosis at $80 \%$, and sequelae of tuberculosis at $76 \%$. In a UK series of 33 COPD patients treated with NPPV over a 5 -yr period, the probability of continuing ventilation was lower at $43 \%$ [41], which is comparable to survival achieved with LTOT $[1,2]$. However, patients included in this series were at the severe end of the spectrum of chronic respiratory failure and most were deteriorating despite LTOT. It is likely that NPPV was instituted too late in the natural history of the condition to achieve optimum benefit.

A study of NPPV in COPD patients who were unable to tolerate LTOT showed that survival was similar to those able to take oxygen therapy and better than historical controls [42]. Factors predicting a poor survival included a high overnight transcutaneous $\mathrm{CO}_{2}$ tension, again emphasising that control of nocturnal hypoventilation is an important factor in the use of NPPV in hypercapnic COPD. Another recent small uncontrolled study of NPPV in COPD found that hospital admissions and primary care consultations were halved with NPPV, although this type of analysis is subject to bias as the use of NPPV involves more supervision and hospital visits [43]. A recent study assigned patients with hypercapnic COPD to two groups depending on whether or not they were able to tolerate NPPV. Although there was no difference in mortality between the two groups, patients taking NPPV had a reduction in intensive care admissions compared to those on LTOT alone [44]. However, this study has some important limitations, as patients were not randomised and only selected on ability to tolerate NPPV. The results of randomised controlled trials, comparing long term outcome of NPPV to oxygen therapy, are awaited.

\section{Recommendations for the use of noninvasive positive- pressure ventilation in chronic obstructive pulmonary disease}

Recommendations for the use of NPPV in COPD are shown in table 1. Results from randomised studies described above suggest that there is now sufficient evidence for the use of NPPV in selected patients with COPD [11]. However, there is no evidence from controlled trials that NPPV in hypercapnic COPD improves survival. Patients must have daytime hypercapnia and documented nocturnal hypoventilation that can be controlled with the chosen ventilator. Patients with the most effective reduction of hypercapnia during the night will be expected to achieve most benefit from the addition of NPPV [11]. Thus, prior to establishment of a patient on home NPPV, investigation with overnight monitoring of hypoventilation and the response to NPPV is required. COPD patients with hypercapnia and considerable sleep disruption will be particularly suitable for long-term ventilation as sleep quality has been shown to improve with NPPV. Obstructive sleep apnoea may coexist with COPD and must be assessed prior to prescribing home NPPV.

Initiation of NPPV for COPD on an inpatient basis may improve compliance $[10,11]$, although this will increase treatment costs. Compliance is frequently affected by complications related to the nasal mask used for ventilation. Although nasal mask complications are common at the start of ventilation, most resolve with specific attention [45]. Early identification of nasal mask complications and intervention will

Table 1.-Noninvasive positive-pressure ventilation (NPPV) in chronic obstructive pulmonary disease: selection criteria of patients for long-term therapy

Established on long-term oxygen therapy

Daytime hypercapnia

End stage disease excluded

Blood gas tensions stable

Evidence of nocturnal hypoventilation

Poor sleep quality with frequent arousals

Nocturnal hypoventilation confirmed to be controlled by NPPV

Appropriate support and education for patient Adequate patient motivation 
improve compliance and thus efficiency of NPPV. Patients chosen for home NPPV must be appropriately motivated and prepared to accept the ventilator at home. An acclimatisation period to ventilation may allow careful adjustments of the settings to increase patient comfort. In some patients with COPD, the presence of intrinsic positive end-expiratory pressure may produce asynchrony between the ventilator and the patient's respiratory efforts [46]. Care must be taken during the initiation of NPPV to ensure adequate capture of ventilation. Patients will be monitored with blood gases and adjustments of the ventilator may be required later, especially when the disease eventually progresses. Various types of ventilators are available for NPPV in COPD, and the choice will depend on local experience, cost and patient acceptance. Both volume and pressure-cycled ventilators are effective in patients with hypercapnic COPD [47], and the choice will depend on patient experience and success in ventilation. Equipment for pressure-support ventilation is generally less expensive than that required for assist-control ventilation and has been used in a number of the reported studies.

The point in the natural history of hypercapnic COPD at which treatment with NPPV should be commenced has not been evaluated. Although LTOT improves survival in patients with hypoxaemic COPD, eventually patients die with progressive irreversible airflow obstruction, with blood gases deteriorating before death [48]. Studies that showed benefit of NPPV in COPD recruited mainly stable patients. However, patients are often referred for further management when blood gases are deteriorating, despite optimum management and LTOT. A study of nine patients with advanced hypercapnic COPD and hypoxaemia refractory to oxygen therapy $\left(\mathrm{Pa}, \mathrm{O}_{2}<\right.$ $8.0 \mathrm{kPa}(60 \mathrm{mmHg})$ on supplemental oxygen $)$ has been reported by Meecham Jones and Wedzicha [49]. The addition of chronic NPPV to LTOT in these patients produced no improvement in clinical condition or blood gases. This suggests that chronic NPPV should be used at an earlier stage in the natural history of the condition, before rapid clinical deterioration occurs. However, several studies demonstrate that patients with acute respiratory failure due to COPD are successfully treated with NPPV [50-52]. Further studies are required to assess the specific subgroup and the optimum time for starting longterm NPPV in patients with COPD. A recently published review [53] about noninvasive ventilation proposes the indications shown in patients with COPD (table 2); these remain to be assessed by prospective clinical trials.

\section{Bronchiectasis}

Patients with bronchiectasis may be difficult to distinguish in clinical practice from patients with COPD. Pulmonary function tests will show an obstructive defect, with or without a restrictive pattern. With disease progression, there is worsening of the ventilation/perfusion ratio and the development of hypoxaemia. Later, the patients will develop
Table 2.-Possible indications for long-term noninvasive positive-pressure ventilation in patients with chronic obstructive pulmonary disease

Exclude possible associated OSAS

Propose NIV if

Symptoms of nocturnal hypoventilation: fatigue, dyspnoea, morning headache

And at least one of

$P \mathrm{a}, \mathrm{CO}_{2} \geqslant 7.3 \mathrm{kpA}$

$\mathrm{Pa}_{\mathrm{a}} \mathrm{CO}_{2}$ 6.7-7.2 kpA and nocturnal oxygen desaturation ( $\leqslant 88 \%$ during 5 consecutive min with $\mathrm{O}_{2} \geqslant 2 \mathrm{~L} \cdot \mathrm{min}^{-1}$ )

$P \mathrm{a}, \mathrm{CO}_{2} 6.7-7.2 \mathrm{kpA}$ and number of hospitalisations due to episodes of acute hypercapnic decompensation $\geqslant 2$ during the 12 past months.

OSAS: obstructive sleep apnoea syndrome; NIV: noninvasive ventilation; $P \mathrm{a}, \mathrm{CO}_{2}$ : carbon dioxide tension in arterial blood; $\mathrm{O}_{2}$ : oxygen. Information taken from [53].

hypoventilation with hypercapnia, although at this stage there is often severe "end stage" lung disease. In addition to the progressive lung disease, patients with bronchiectasis have copious sputum production and recurrent infection, which cause further problems during assisted ventilation and weaning [54].

In the French series on patients using positivepressure ventilation via tracheostomy, survival was inferior for those with bronchiectasis, compared to patients with COPD and chest wall deformity or neuromuscular disease [27]. In a series of patients with bronchiectasis and respiratory failure treated by NPPV recently reported in the UK, the major outcome measure was probability of continuing NPPV, which was $<20 \%$ after 2 yrs [41]. The NPPV was started late in the natural history of the condition and the patients became increasingly ventilatordependent during the treatment period. All the patients with bronchiectasis had been referred for consideration of heart/lung transplantation. In the study by LEGER et al. [40], 25 patients were treated with NPPV for bronchiectasis. There was a higher probability of continuing NPPV than in the British study. However, significant changes in blood gases were not seen and in contrast to the other indications for NPPV, there was no reduction observed in days of hospitalisation for respiratory illness. One retrospective study of NPPV using volume-cycled ventilators in bronchiectasis, found that although the $\mathrm{Pa}, \mathrm{CO}_{2}$ was not reduced, it was stabilised with NPPV and that the number of days in hospital was reduced [54]. Another retrospective, case-controlled study of 14 severe patients with severe bronchiectasis and chronic respiratory failure showed a satisfactory long-term tolerance and compliance in 11 , with a beneficial effect on hospitalisation rate and functional status, but no effect on survival [55].

\section{Cystic fibrosis}

Patients with cystic fibrosis who are intubated may have a prolonged clinical course in the intensive care unit and considerable difficulties with weaning. The availability of heart/lung transplantation provides hope to end-stage patients with cystic fibrosis and 
progressive respiratory failure, as such patients may be removed from transplantation lists if intubated. NPPV has been used in the management of these patients in an effort to avoid complications of intubation and to serve as a bridge to transplantation [56].

PIPER et al. [57] treated four patients with cystic fibrosis, who had hypercapnic respiratory failure for up to 18 months. Within a few days of starting NPPV, hypercapnia was reduced and patients reported improved length and quality of sleep. After stabilisation, all the patients were discharged home, with improvements maintained for up to 18 months. HiLL et al. [58] followed 10 patients with cystic fibrosis awaiting transplantation who were treated with home NPPV for up to 15 months, NPPV being used as a bridge to lung transplantation. At 3 months after treatment they observed improvements in forced vital capacity, $\mathrm{Pa}, \mathrm{CO}_{2}$ and also a significant reduction in the number of hospital inpatient days. However, in this study patients were generally started on NPPV late in the natural history of their disease, though earlier use may have advantages and requires study.

\section{Conclusion}

Noninvasive positive-pressure ventilation, when used in combination with domiciliary long-term oxygen therapy in selected stable chronic obstructive pulmonary disease patients with hypercapnic respiratory failure, can control hypoventilation and this is associated with improved daytime arterial blood gas tensions, sleep and quality of life. The patients who show the greatest reduction in overnight carbon dioxide tension in arterial blood with ventilation are most likely to benefit from long-term ventilatory support. Although there is now evidence for short-term benefit of noninvasive positive-pressure ventilation in hypercapnic chronic obstructive pulmonary disease, large multicentre studies with survival, exacerbations and hospital admissions as the primary end points are required to evaluate longer term effects of this potentially important intervention. Current data suggest a possible role, particularly as a bridge to transplantation in patients with bronchiectasis and cystic fibrosis, but again further data are needed to establish whether earlier intervention is indicated.

\section{References}

1. Nocturnal Oxygen Therapy Trial Group. Continuous or nocturnal oxygen therapy in hypoxaemic chronic obstructive lung disease. Ann Intern Med 1980; 93: 391-398.

2. Medical Research Council Working Party. Long term domiciliary oxygen therapy in chronic hypoxic cor pulmonale complicating chronic bronchitis and emphysema. Lancet 1981; i: 681-686.

3. Begin P, Grassino A. Inspiratory muscle dysfunction and chronic hypercapnia in chronic obstructive pulmonary disease. Am Rev Respir Dis 1991; 143: 905-912.

4. Douglas NJ, Calverley PMA, Leggett RJE, Brash HM,
Flenley DC, Brezinova V. Transient hypoxaemia during sleep in chronic bronchitis and emphysema. Lancet 1979; i: 1-4.

5. Wynne JW, Block AJ, Hemenway J, Hunt LA, Flick MR. Disordered breathing and oxygen desaturation during sleep in patients with chronic obstructive lung disease (COLD). Am J Med 1979; 66: 573-579.

6. Celli B, Lee $\mathrm{H}$, Criner $\mathrm{G}$, et al. Controlled trial of external negative pressure ventilation in patients with severe airflow limitation. Am Rev Respir Dis 1989; 140: 1251-1256.

7. Shapiro SH, Ernst P, Gray-Donald K, et al. Effect of negative pressure ventilation in severe chronic obstructive pulmonary disease. Lancet 1992; 340: 1425-1429.

8. Zibrak JD, Hill NS, Federman EC, Kwa SL, $\mathrm{O}^{\prime}$ Donnell C. Evaluation of intermittent long-term negative-pressure ventilation in patients with severe chronic obstructive pulmonary disease. Am Rev Respir Dis 1988; 138: 1515-1518.

9. Carroll N, Branthwaite MA. Control of nocturnal hypoventilation by nasal intermittent positive pressure ventilation. Thorax 1988; 43: 349-353.

10. Elliott MW, Simonds AK, Carroll MP, Wedzicha JA, Branthwaite MA. Domiciliary nocturnal nasal positive pressure ventilation in hypercapnic respiratory failure due to chronic obstructive lung disease: effects on sleep and life quality. Thorax 1992; 47: 342-348.

11. Meecham Jones DJ, Paul EA, Jones PW, Wedzicha JA. Nasal pressure support ventilation plus oxygen compared to oxygen therapy alone in hypercapnic COPD. Am J Respir Crit Care Med 1995; 152: 538 544.

12. Douglas NJ. Sleep. In: Calverley $\mathrm{P}$, Pride N, eds. Chronic obstructive pulmonary disease. London, Chapman \& Hall, 1995; pp. 293.

13. Ingrassia RS, Nelson SB, Harris CD, Hubmayr RD. Influence of sleep state on $\mathrm{CO}_{2}$ responsiveness. $\mathrm{Am}$ Rev Respir Dis 1991; 144: 1125-1129.

14. Weigland L, Zwillich CW, White DP. Sleep and the ventilatory response to resistive loading in normal men. J Appl Physiol 1988; 64: 1186-1195.

15. Goldstein RS, Ramcharan V, Bowes G, McNicholas WT, Bradley D, Phillipson EA. Effect of supplemental nocturnal oxygen on gas exchange in patients with severe obstructive lung disease. N Engl J Med 1984; 310: 425-429.

16. Fletcher EC, Scott D, Qian W, Luckett RA, Miller CC, Goodnight-White S. Evolution of nocturnal oxyhemoglobin desaturation in patients with chronic obstructive pulmonary disease and a daytime $\mathrm{PaO}_{2}$ above 60 mmHg. Am Rev Respir Dis 1991; 144: 401405.

17. Juan G, Calverley P, Talamo C, Schnader J, Roussos C. Effect of carbon dioxide on diaphragmatic function in human beings. $N$ Engl J Med 1984; 310: 874-879.

18. Belman MJ, Soo Hoo GW, Kuei JH, Shadmehr R. Efficacy of positive $v s$ negative pressure ventilation in unloading the respiratory muscles. Chest 1990; 98: $850-856$.

19. Carrey Z, Gottfried SB, Levy RD. Ventilatory muscle support in respiratory failure with positive pressure ventilation. Chest 1990; 97: 150-158.

20. Renston JP, DiMarco AF, Supinski GS. Respiratory muscle rest using nasal BiPAP ventilation in patients with stable severe COPD. Chest 1994; 105: 1053-1060.

21. Hill NS, Eveloff SE, Carlisle CC, Goff SG. Efficacy of nocturnal nasal ventilation in patients with restrictive 
thoracic disease. Am Rev Respir Dis 1992; 145: 365 371.

22. Goldstein RS, Molotiu N, Skrastins R, et al. Reversal of sleep induced hypoventilation and chronic respiratory failure by nocturnal negative pressure ventilation in patients with restrictive ventilatory impairment. $\mathrm{Am}$ Rev Respir Dis 1987; 135: 1049-1055.

23. Elliott MW, Mulvey DA, Moxham J, Green M, Branthwaite MA. Domiciliary nocturnal nasal intermittent positive pressure ventilation in COPD: mechanisms and underlying changes in arterial blood gas tensions. Eur Respir J 1991; 4: 1044-1052.

24. Calverley PMA, Brezinova V, Douglas NJ, Catterall JR, Flenley DC. The effect of oxygenation on sleep quality in chronic bronchitis and emphysema. Am Rev Respir Dis 1982; 126: 206-210.

25. Brezinova V, Catterrall JR, Douglas NJ. Night sleep of patients with chronic ventilatory failure and agematched controls. Number and duration of EEG episodes of intervening wakefulness and drowsiness. Sleep 1982; 5: 123-130.

26. Hedemark L, Kronenberg R. Ventilatory responses to hypoxia and $\mathrm{CO}_{2}$ during natural and flurazepam induced sleep in normal adults. Am Rev Respir Dis 1981; 123: 190 .

27. Robert D, Gerard M, Leger $\mathrm{P}$, et al. La ventilation mechanique a domicile definitive par tracheostomie de l'insuffisant respiratoire chronique. Rev Mal Respir 1983; 11: 923-936.

28. Muir JF, Girault C, Cardinaud JP, Polu JM and and Cooperative group. Multicenter study of 259 severe COPD patients with tracheostomy and home ventilation. Chest 1994; 105: 100-105.

29. Gutierrez M, Beroiza T, Contreras G, et al. Weekly cuirass ventilation improves blood gases and inspiratory muscle strength in patients with chronic airflow limitation and hypercarbia. Am Rev Respir Dis 1988; 138: 617-623.

30. Cropp A, DiMarco AF. Effects of intermittent negative positive pressure ventilation on respiratory muscle function in patients with severe chronic obstructive pulmonary disease. Am Rev Respir Dis 1987; 135: 1056-1061

31. Marino W. Intermittent volume cycled mechanical ventilation via nasal mask in patients with respiratory failure due to COPD. Chest 1991; 99: 681-684.

32. Strumpf DA, Millman RP, Carlisle CC, et al. Nocturnal positive-pressure ventilation via nasal mask in patients with severe chronic obstructive pulmonary disease. Am Rev Respir Dis 1991; 144: 1234-1239.

33. McSweeney AJ, Grant JA, Heaton RK, Adams KM, Timms RM. Life quality of patients with chronic obstructive pulmonary disease. Arch Intern Med 1982; 142: 473-478.

34. Okubadejo AA, Paul EA, Jones PW, Wedzicha JA. Does long term oxygen therapy affect quality of life in patients with chronic obstructive pulmonary disease and severe hypoxaemia? Eur Respir J 1996; 9: 2335 2339.

35. Jones PW, Quirk FH, Baveystock CM, Littlejohns P. A self-complete measure of health status for chronic airflow limitation. Am Rev Respir Dis 1992; 145: 13211327.

36. Pehrsson K, Olofson J, Larsson S, Sullivan M. Quality of life of patients treated by home mechanical ventilation due to restrictive ventilatory disorders. Respir Med 1994; 88: 21-26.
37. Seemungal TAR, Donaldson GC, Paul EA, Bestall JC, Jeffries DJ, Wedzicha JA. Effect of exacerbation on quality of life in patients with chronic obstructive pulmonary disease. Am J Respir Crit Care Med 1998; 157: $1418-1422$.

38. Perrin C, El Far Y, Vandenbos F, et al. Domiciliary nasal intermittent positive pressure ventilation in severe COPD: effects on lung function and quality of life. Eur Respir J 1997; 10: 2835-2839.

39. Lin C-C. Comparison between nocturnal nasal positive pressure ventilation combined with oxygen therapy and oxygen monotherapy in patients with severe COPD. Am J Respir Crit Care Med 1996; 154: 353-358.

40. Leger P, Bedicam JM, Cornette A, et al. Nasal intermittent positive pressure ventilation. Long term follow-up in patients with severe chronic respiratory insufficiency. Chest 1994; 105: 100-105.

41. Simonds AK, Elliott MW. Outcome of domiciliary nasal intermittent positive pressure ventilation in restrictive and obstructive disorders. Thorax 1995; 50: 604-609.

42. Sivasothy P, Smith IE, Shneerson JM. Mask intermittent positive pressure ventilation in chronic hypercapnic respiratory failure due to chronic obstructive pulmonary disease. Eur Respir J 1998; 11: 3440.

43. Jones SE, Packham, Hebden M, Smith AP. Domiciliary nocturnal intermittent positive pressure ventilation in patients with respiratory failure due to severe COPD: long term follow up and effect on survival. Thorax 1998; 53: 495-498.

44. Clini E, Sturani C, Porta R, et al. Outcome of COPD patients performing nocturnal non-invasive mechanical ventilation. Respir Med 1998; 92: 1215-1222.

45. Meecham Jones DJ, Wedzicha JA. Nasal masks for positive pressure ventilation: survey of patient usage and complications. Thorax 1994; 49: 811-812.

46. Elliott MW, Mulvey DA, Moxham J, Green M, Branthwaite MA. Inspiratory muscle effort during nasal intermittent positive pressure ventilation in patients with chronic obstructive airways disease. Anaesthesia 1993; 48: 8-13.

47. Meecham Jones DJ, Wedzicha JA. Comparison of pressure and volume preset nasal ventilator systems in stable chronic respiratory failure. Eur Respir J 1993; 6: $1060-1064$.

48. Cooper CB, Howard P. An analysis of sequential physiologic changes in hypoxic cor pulmonale during long term oxygen therapy. Chest 1991; 100: 76-80.

49. Meecham Jones DJ, Wedzicha JA. Non-invasive positive pressure ventilation in advanced progressive chronic respiratory failure due to COPD. Am Rev Respir Dis 1993; 147: A322.

50. Bott J, Carroll MP, Conway JH, et al. Randomised controlled trial of nasal ventilation in acute ventilatory failure due to chronic obstructive airways disease. Lancet 1993; 341: 1555-1557.

51. Brochard L, Mancero J, Wysocki M, et al. Noninvasive ventilation for acute exacerbations of chronic obstructive pulmonary disease. $N$ Engl J Med 1995; 333: 817-822.

52. Kramer N, Meyer TJ, Meharg J, Cece RD, Hill NS. Randomised prospective trial of noninvasive positive pressure ventilation in acute respiratory failure. $\mathrm{Am}$ J Respir Crit Care Med 1995; 151: 1799-1806.

53. Clinical indications for non invasive positive pressure ventilation in chronic respiratory failure due 
to restrictive lung disease, COPD, and nocturnal hypoventilation-a consensus conference report. Chest 1999; 116: 521-534.

54. Gacouin A, Desrues B, Lena H, Quinquenel ML, Dassonville J, Delaval P. Long term nasal intermittent positive pressure ventilation (NIPPV) in sixteen consecutive patients with bronchiectasis: a retrospective study. Eur Respir J 1996; 9: 1246-1250.

55. Benhamou D, Muir JF, Raspaud C, et al. Long term efficiency of home nasal mask ventilation in patients with diffuse bronchectasis and severe chronic respiratory failure: a case-control study. Chest 1997; 112: 1259-1266.
56. Hodson ME, Madden BP, Steven MH, Tsang VT, Yacoub MH. Non-invasive mechanical ventilation for cystic fibrosis patients-a potential bridge to transplantation. Eur Respir J 1991; 4: 524-527.

57. Piper AJ, Parker S, Torzillo PJ, Sullivan CE, Bye PTB. Nocturnal nasal IPPV stabilizes patients with cystic fibrosis and hypercapnic respiratory failure. Chest 1992; 102: 846-850.

58. Hill AT, Edenborough FP, Cayton RM, Stableforth DE. Long-term nasal intermittent positive pressure ventilation in patients with cystic fibrosis and hypercapnic respiratory failure. Respir Med 1998; 92: 523-526. 